\title{
PLANET-B magnetic fields investigation
}

\author{
T. Yamamoto and A. Matsuoka \\ Institute of Space and Astronautical Science, Sagamihara, Kanagawa 229-8510, Japan
}

(Received August 13, 1997; Revised November 18, 1997; Accepted December 2, 1997)

\begin{abstract}
Some scientific topics relating to magnetic field measurements around Mars are briefly described and specification of the magnetic field instrument onboard PLANET-B is presented. The highly elliptical orbit of PLANET-B with a periapsis altitude of $150 \mathrm{~km}$ and an apoapsis of $15 \mathrm{R}_{\mathrm{M}}$ (Mars radii) will enable us to observe magnetic environments in various regions from the ionospheric altitudes to upstream and tail regions. One of the most important objectives is to resolve the controversial arguments about the magnitude of the magnetic moment of Mars. Combination of measurements with other instruments onboard PLANET-B will give us important information on unsolved problems such as the pressure balance in the dayside ionosphere and acceleration processes for ionospheric ions.
\end{abstract}

\section{Introduction}

The primary scientific mission of PLANET-B aims at the understanding of the physics of the solar wind-upper atmosphere interaction around the planet Mars. The Martian upper atmosphere remains unexplored with in-situ measurements in spite of many efforts, except for the two Viking landers. This is in great contrast to Venus, the upper atmosphere of which was extensively observed with the Pioneer Venus Orbiter (PVO) spacecraft. PLANET-B will be inserted into an elliptical orbit around Mars in October 1999. The periapsis altitude is about $150 \mathrm{~km}$ and the apoapsis is about 15 Mars radii. This orbit enables us to observe the upper atmosphere and the ionosphere at lower altitudes. We can also obtain accelerated ionospheric species in the nightside tail region with this orbit. Bow shock and upstream regions are also within the scope of the PLANET-B mission. The magnetic field instrument will contribute to the mission by measuring magnetic field vectors in the solar wind, magnetosheath, ionosphere, and magnetotail with a high time resolution.

The magnetic environment of Mars is still unknown although many spacecraft have been sent there in the past. Most of the previous missions aiming to explore the upper atmosphere of Mars did not operate long enough to collect a reasonable amount of data to produce convincing studies about the Martian upper atmosphere, except for the successful Viking mission that did not carry a magnetic field instrument. Phobos 2 carried a magnetometer and its closest approach was about $850 \mathrm{~km}$. Since Phobos 2 was operating in the Mars orbit for only 2 months, the amount of magnetic field data from that mission is not enough to figure out the magnetic field environments of the planet. The lack of low altitude

Copy right $(\subset)$ The Society of Geomagnetism and Earth, Planetary and Space Sciences (SGEPSS); The Seismological Society of Japan; The Volcanological Society of Japan; The Geodetic Society of Japan; The Japanese Society for Planetary Sciences. measurements of magnetic fields, makes it especially difficult to model the upper atmosphere of Mars and its interaction with the solar wind. Mars has a steady ionospheric structure. Its peak density is about $10^{5} \mathrm{~cm}^{-3}$ and a peak height is about 150 km (e.g., Zhang, M. H. G. et al., 1990). Large ionospheric electric currents are expected and should modify the magnetic field configuration significantly around the topside ionosphere, even if Mars has an intrinsic magnetic field in the order of $50 \mathrm{nT}$ at ionospheric altitudes. High resolution measurements of magnetic fields at lower altitudes, below the ionospheric current layer if possible, are quite necessary. Since the periapsis altitude is quite low (about $150 \mathrm{~km}$ ), the PLANET-B magnetic field experiment surely addresses the questions.

There are many questions relating to magnetic field structures around Mars, such as the presence of an intrinsic magnetic field, location and shape of bow shock, and configuration of the draping field that forms the Martian magnetotail. In this paper, we point out some scientific topics relating to the magnetic field measurements onboard PLANET-B. The fundamental function of the PLANET-B magnetic field equipment (MGF) is also briefly described.

\section{Scientific Topics Relating to Magnetic Field Measurements}

In magnetized plasmas, the magnetic field plays an essential role in delineating a global structure and dynamical processes taking place there. In this regard, magnetic field measurements are a key observation in understanding the physical processes in any places where plasmas are dominant constituents. In the Martian upper atmosphere, plasmas are important in characterizing its environment and the magnetic field should control the motion and energy transfer of plasmas there. Without magnetic field data, interpretation of observed data from other instruments onboard PLANETB becomes very difficult. Here, we point out some of the scientific topics relating to magnetic field measurements around Mars. 


\subsection{Intrinsic and crustal field}

The magnitude of the Martian intrinsic magnetic field is still unknown. The determination of the magnetic moment is one of the most important objectives in the PLANET-B mission. There has been much controversy concerning the magnitude of the magnetic moment of Mars (Russell, 1993). This is actually because no spacecraft carrying magnetometers have measured magnetic fields at low altitudes. Before the recent arrival of the Mars Global Surveyor at Mars, the closest approach was made by the Phobos 2 mission. Its lowest height was about $850 \mathrm{~km}$, and is far above the major current layer in the ionosphere. Estimation of the magnetic moment depends on the interpretation of the observed magnetic fields. Dolginov et al. $(1973,1976)$ assumed that the magnetic fields observed with Mars 2, Mars 3 and Mars 5 spacecraft were influenced by an intrinsic magnetic field of Mars and concluded that the magnetic moment was of $2.4-2.5 \times 10^{22} \mathrm{G} \cdot \mathrm{cm}^{3}$. On the other hand, Russell (1978a, b) claimed that magnetic fields observed by Mars-series spacecraft tend to be wrapped around the planet, and proposed an upper limit to the magnetic moment of $2 \times 10^{21} \mathrm{G} \cdot \mathrm{cm}^{3}$. Russell et al. (1995) showed that the direction of the magnetic field in the tail was highly correlated with the direction of the interplanetary magnetic field (IMF) and estimated the upper limit to the magnetic moment as $4 \times 10^{21} \mathrm{G} \cdot \mathrm{cm}^{3}$. There is still more than one order of magnitude difference in the estimated magnetic moment. Recently, it was reported that the magnetic field is likely to be of crustal rather than of dynamo origin, based on the Mars Global Surveyor data (Showstack, 1997). The field varied in strength at different locations, and anomalous magnetic fields of up to $400 \mathrm{nT}$ were observed.

The strength of the internal magnetic fields at the topside ionosphere would relate to the fundamental features of the interaction between the solar wind and the Martian ionosphere. Under a typical solar wind condition around Mars (number density $\sim 2.5 \mathrm{~cm}^{-3}$ and speed $\sim 400 \mathrm{~km} / \mathrm{s}$ ), a magnetic field strength of about $40 \mathrm{nT}$ could stand off the solar wind. Magnetic moments of $2.4 \times 10^{22} \mathrm{G} \cdot \mathrm{cm}^{3}$ and $2 \times 10^{21}$ $\mathrm{G} \cdot \mathrm{cm}^{3}$ would produce about 60 and $5 \mathrm{nT}$, respectively, at the topside ionosphere. From the range of previous estimations of the magnetic field, the nature of the interaction of the solar wind with the upper atmosphere of Mars is quite ambiguous. An intrinsic magnetic field might stop the solar wind around the topside ionosphere or, especially during solar minimum, the Martian ionosphere might be compressed and the altitude of the upper boundary might decrease down to $200 \mathrm{~km}$ (Zhang and Luhmann, 1992). In any case, the determination of the magnetic moment from an observational basis is quite essential. Low altitude $(\sim 150 \mathrm{~km})$ measurements of the magnetic field with PLANET-B will surely resolve this problem. Figure 1 shows the PLANET-B orbital track at lower altitudes $(<1000 \mathrm{~km})$. Although the low altitude observations are biased to the southern hemisphere as a whole, nightside observations when the solar zenith angle is larger than $90^{\circ}$ are well distributed from $-40^{\circ}$ to $20^{\circ}$. Since the solar wind effect is the least significant in the nightside ionosphere, PLANET-B magnetic field measurements will be most important in resolving the controversial arguments about the magnetic moment of Mars.

The magnitude of the magnetic moment also gives us important information on the internal structure of Mars. The presence of a substantial magnetic moment could indicate that a planetary dynamo action in the Martian core is working. This will impose a bounding condition to the thermal history of Mars.

In addition to a planetary scale magnetic field structure that would indicate a presence or absence of the Martian intrinsic magnetic field, we might also expect coherent signals with a moderate spatial scale due to crustal magnetic anomalies. The detection of a crustal magnetic field is another important issue relating to the intrinsic magnetic field in the past. The high oxidation state expected for the Martian surface suggests that Martian crustal magnetization may be large indeed. Volcanic structures and craters produced in the past may have acquired magnetization due to an ancient magnetic field, if it existed. The low periapsis altitude of PLANET-B has again a big advantage in detecting the crustal magnetic field. Simultaneous observations

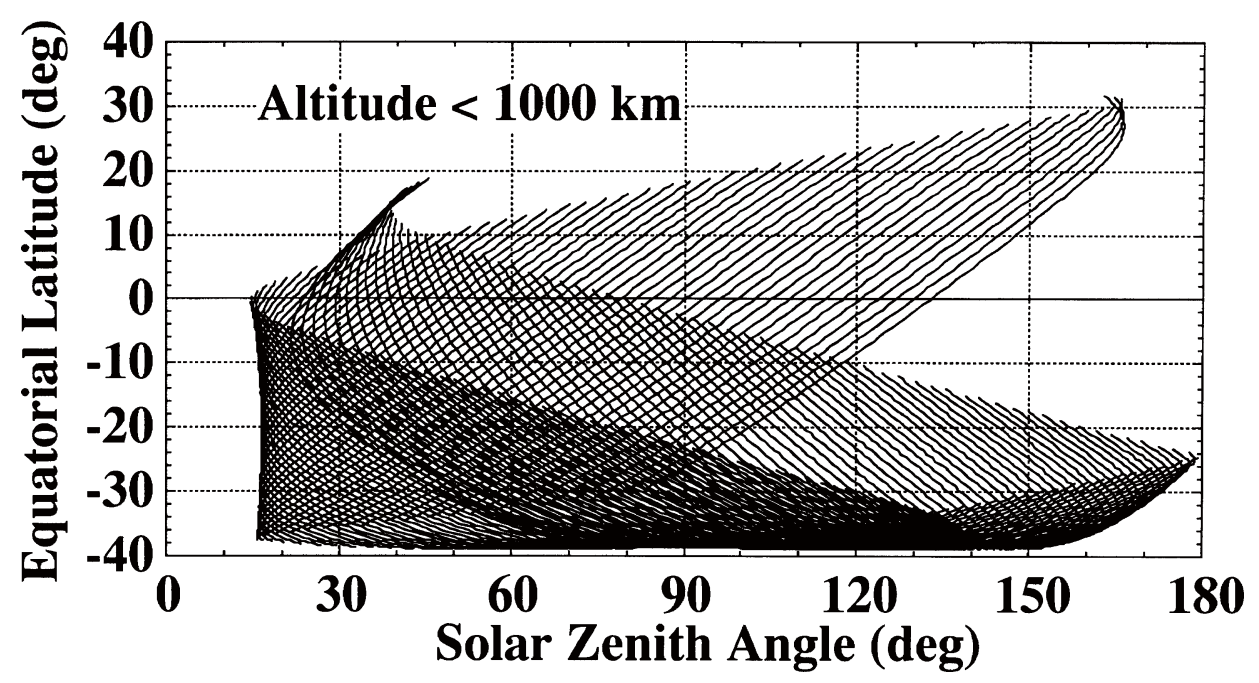

Fig. 1. Orbit tracks of PLANET-B when the altitude is below $1000 \mathrm{~km}$. The horizontal axis is the solar zenith angle at the spacecraft location and the vertical axis is the equatorial latitude of Mars. 
with the visible camera (MIC) will reveal magnetic anomalies relating to the surface structures and PLANET-B may figure out the history of the magnetic moment of Mars. If we can really do this, the results would give important information on the evolution of the internal structure of Mars.

\subsection{Global ionospheric current structure}

When the solar wind interacts with ionospheric plasmas, it gives a part of its momentum through various physical processes, and eventually drives a global ionospheric current. The resultant ionospheric current, in turn, produces a global magnetic field structure at the ionospheric altitudes which contributes to stand off the dynamic pressure of the solar wind. A weak (or nonexistent) intrinsic magnetic field implies that the ionospheric current is highly variable in time and in space, especially in the dayside region. If the intrinsic magnetic field is absent, the ionospheric current would be totally controlled by the solar wind and the interplanetary magnetic field. If a small magnitude of an intrinsic magnetic field is present, the situation could be much more complicated.

There are at least three driving mechanisms for the ionospheric electric current. They are, thermospheric wind, viscous interaction at a boundary that separates the solar wind region and the ionosphere, and solar wind-ionosphere coupling through draped magnetic field lines. Since the ionospheric plasma pressure is not large enough to stand off the solar wind (Luhmann and Brace, 1991), there should be some magnitude of magnetic field in the ionosphere. Shinagawa and Cravens (1989) evaluated about $50 \mathrm{nT}$, based on the assumption that it is induced like that of Venus. With this magnitude of magnetic field, the ionosphere is an anisotropic conductivity media. Hall and Pederson conductivity becomes meaningful. The primary magnetic field in the ionosphere is expected to be horizontal (Luhmann and Brace, 1991). Suppose the main driving force for the ionospheric current is the viscous interaction at the topside ionosphere. The driving force generates a large-scale ionospheric plasma circulation and the resultant electric field is in the vertical direction. With this electric field, Hall current flows in the horizontal plane so as to shield the interplanetary magnetic field, while Pederson current flows in the vertical direction.

A small magnitude of intrinsic magnetic field would make the situation much more complicated. The pattern of ionospheric current would be partly controlled by the solar wind conditions, but partly restricted by the internal magnetic field. A $50 \mathrm{nT}$ magnetic field could stand off the solar wind, but the stand-off distance is so close to (or inside when a solar wind dynamic pressure is relatively large) the topside ionosphere. Since the ionosphere is collisional media, magnetic reconnection processes could be operating between the interplanetary magnetic field (IMF) and the intrinsic field when the direction of the IMF is antiparallel to the internal magnetic field. If this is the case, a substantial amount of ionospheric particles would be accelerated and escape out with the solar wind under reconnection processes.

Figure 2 shows the PLANET-B orbit tracks when its altitude is lower than $1000 \mathrm{~km}$. PLANET-B will cover a wide area of ionospheric heights with a solar zenith angle from $15^{\circ}$ to $170^{\circ}$. This orbit coverage will enables us to survey the global ionospheric current patterns of Mars.

\subsection{Solar wind-ionosphere interactions}

One of the important issues still unsolved in the Martian upper atmosphere environment is the pressure balance of plasmas in the dayside area. During solar maximum, the thermal pressure of the ionosphere could balance with the solar wind pressure at altitudes above $300 \mathrm{~km}$ (Zhang and Luhmann, 1992). On the other hand, the thermal pressure during solar minimum estimated from Viking lander observations shows that the ionospheric pressure alone cannot sustain the dynamic pressure of the solar wind (Luhmann and Brace, 1991). The evidence indicates that another contribution, most probably from magnetic pressure, is needed to interpret the height profile of ionospheric electron density. Anyway, there should be a magnetic field, either intrinsic or solar-wind induced, in the Martian ionosphere. Measurements of magnetic field strength in the ionospheric altitudes are, thus, very important, together with electron

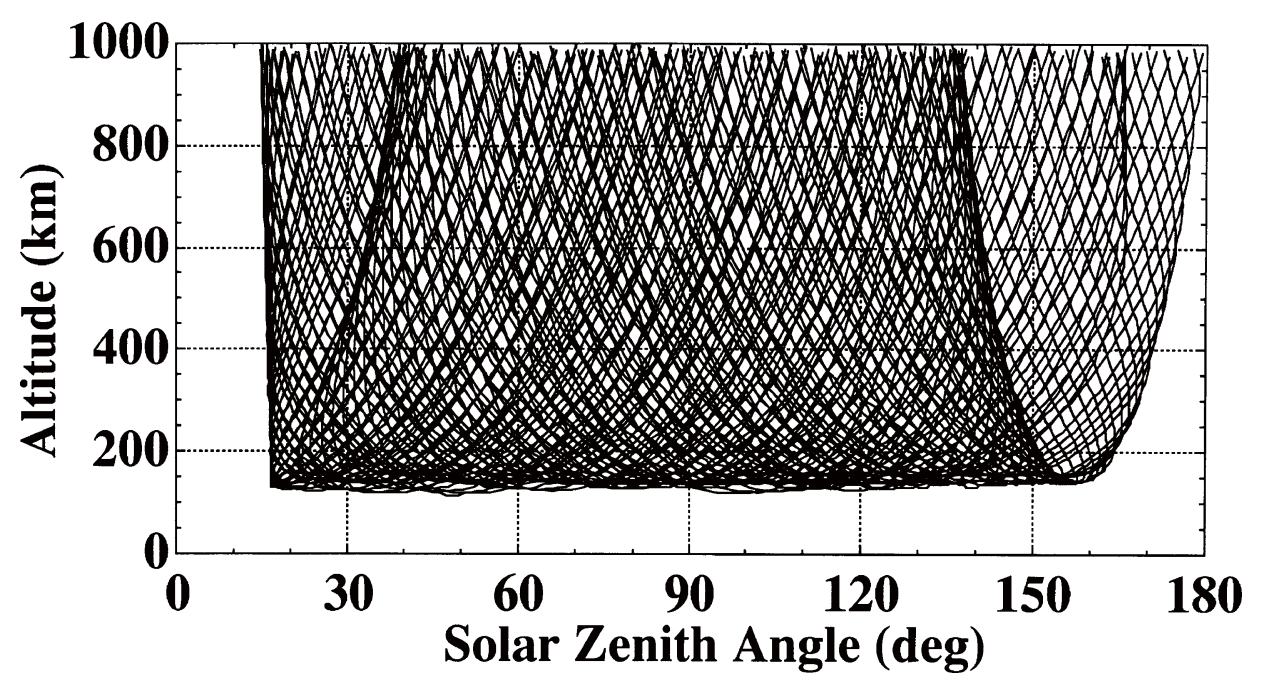

Fig. 2. Orbit tracks of PLANET-B. The horizontal axis is the solar zenith angle at the spacecraft location and the vertical axis is the altitude of the spacecraft. 


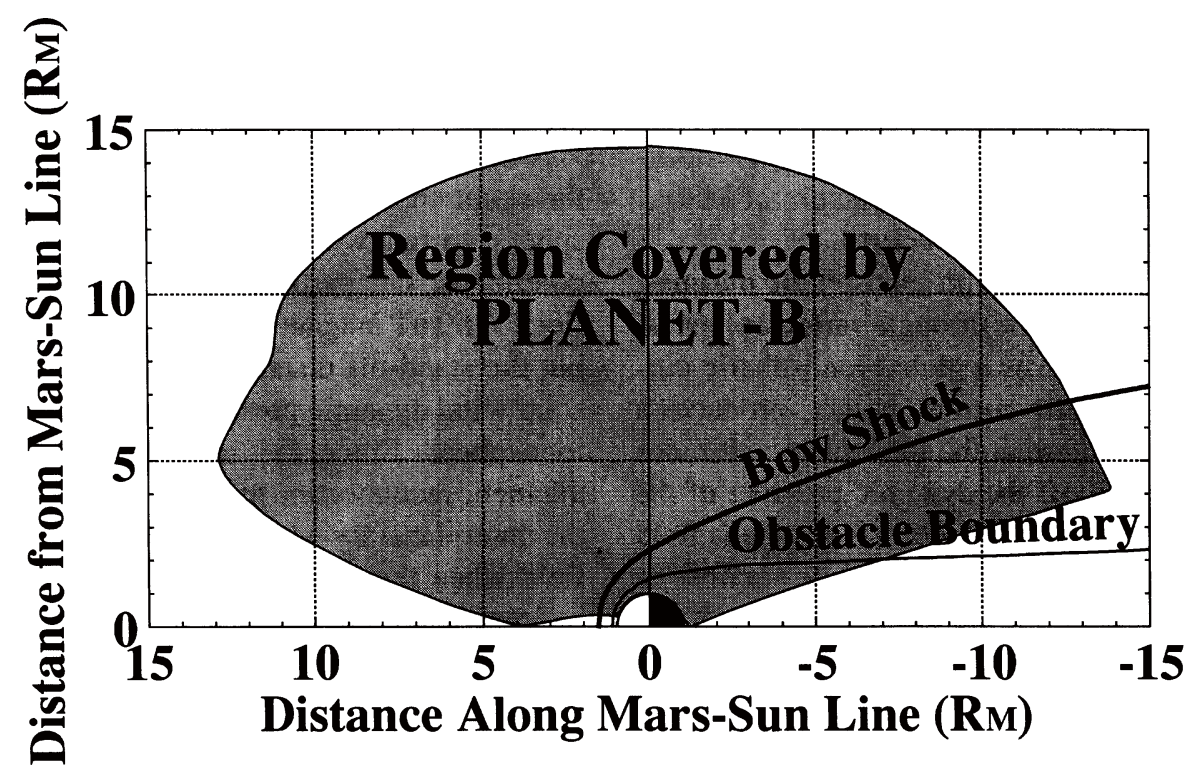

Fig. 3. Orbital coverage of PLANET-B in higher altitudes. The horizontal axis is the distance along the Mars-Sun line and the vertical axis is the distance from the Mars-Sun line. The location of bow shock and obstacle boundary determined from Phobos 2 observations (from Schwingenschuh et al., 1990) are shown.

density (PWS) and electron temperature measurements (PET), in discussing pressure balance problems.

Total pressure of magnetized plasma is represented by the sum of plasma thermal pressure, plasma dynamic pressure, and magnetic pressure. In a steady-state condition, there is no spatial gradient of the total pressure. The variation in contribution of each pressure term along the Mars-Sun line in the dayside region would characterize the region of interest. With this idea, Sauer et al. (1990) proposed three plasma boundaries. They are, from the upstream to the ionosphere, bow shock, planetopause, and magnetopause, although there have been some arguments about the identification of the planetopause. Figure 3 shows an expected orbit coverage of PLANET-B around Mars. The orbit coverage is, of course, not perfect, especially along the MarsSun line, because of the limitation of the maximum eclipse time interval. By gathering data when the spacecraft is close to the Mars-Sun line, we will be able to discuss the pressure balance and boundaries that characterize the interaction between the solar wind and the ionosphere.

The solar wind approaching to Mars will first encounter hot upper atmospheric atoms before reaching the main body of the ionosphere. Some of these atoms will be ionized by charge exchange and/or solar UV in the solar wind region, and be transported with the solar wind toward the tail. This is the ion pick-up process. Its rate has not been experimentally measured at Mars. The pick-up process is known to be controlled by the magnetic field orientation in the solar wind. When the magnetic field vector makes a large angle with the solar wind flow direction, pick-up ions acquire greater energy from the solar wind. The small radius of Mars compared with the gyro-radius of the pick-up ions should produce an asymmetric distribution of energetic ions in the dayside solar wind region and in the nightside magnetotail, where the magnetic field governs the asymmetric axis. The combination of ion measurements with magnetic field data allows us to investigate the pick-up processes around Mars.

The magnetic field in the wake of Mars characterizes the escaping ions from the planet. The configuration of the Martian magnetotail is an important issue in discussing the consequence of the escaping ions and how they are accelerated after capture by the solar wind. Phobos 2 was the first spacecraft to make magnetic field measurements in the deep wake. It was argued that at least near the equatorial plane at a radial distance of about $2.7 \mathrm{R}_{\mathrm{M}}$, the Martian wake field appears to be similar to that in the induced magnetotail of unmagnetized Venus (Yeroshenko et al., 1990). The polarity of the lobes in an induced magnetotail is determined by the interplanetary magnetic field orientation. These observations set an upper limit for the planetary magnetic field of Mars (Russell et al., 1984; Dolginov, 1986).

\subsection{Bow shock and upstream region}

Venusian bow shock locations show clear solar activity dependence (Zhang, T.-L. et al., 1990). For the case in Mars, Russell et al. (1992) argued that the bow shock location depends on a solar activity similar to the Venusian case, by comparing the data from Mars 2, 3, and 5 with the data from Phobos. To the contrary, Slavin et al. (1991) argued that the location of the observed Martian bow shock does not depend on the solar activity and seems to be rather controlled by the dynamic pressure of the solar wind. Slavin's result suggests an Earth-like magnetosphere and again gives rise to the controversial argument for the presence of the Martian intrinsic magnetic field. Slavin et al. (1991) assumed a magnetic moment of $1.4 \times 10^{22} \mathrm{G} \cdot \mathrm{cm}^{3}$ (about $35 \mathrm{nT}$ at the Martian surface) to explain the observed bow shock location. The corresponding obstacle height under a typical solar wind condition ranges between 500 and $2000 \mathrm{~km}$. If Mars has an intrinsic magnetic field such as assumed by Slavin et al. (1991), the location of the magnetopause is very close to the topside ionosphere. The situation is quite different from that of Earth, where the magnetopause is located around 
Table 1. Specification of the PLANET-B magnetic field instrument.

$\begin{array}{ll}\begin{array}{l}\text { Sensor } \\ \text { weight }\end{array} & \text { Ring core geometry, three components } \\ \text { Electronics } & 230 \mathrm{~g} \\ \text { weight } & \\ \text { Drive frequency } & 2.72 \mathrm{~kg} \text { including UVS, PET and MST electronics } \\ \text { Dynamic ranges } & 14.8 \mathrm{kHz} \pm 0.5 \mathrm{kHz} \\ \text { Quantization steps (15 bit resolution) } & \pm 16 \mathrm{nT} ; \pm 256 \mathrm{nT} ; \pm 1024 \mathrm{nT} ; \pm 65536 \mathrm{nT} \\ \text { Sampling rate } & 0.001 \mathrm{nT} ; 0.015 \mathrm{nT} ; 0.063 \mathrm{nT} ; 4 \mathrm{nT} \\ & 256 \text { vectors/spin (spin rate: } 7.5 \mathrm{rpm})\end{array}$

$10 \mathrm{R}_{\mathrm{E}}$ in the dayside, far above the ionosphere. The configuration of the Martian bow shock should be significantly affected by the ionospheric current. A simple analogy of the Earth bow shock might not make sense at Mars.

The gyro-radius of pick-up ions could reach an obstacle size that is comparable to the Mars diameter. Because the obstacle size against the solar wind is small, the bow shock at Mars could be different from that at Earth. The motion of the solar wind ions around the subsolar bow shock location would not be gyrotropic, because they would be lost by impacting the ionosphere. The bow shock at Mars would be diffuse in comparison with those in the other planets of the solar system (the Mercury bow shock might be also diffuse because of its small size). The determination of the bow shock shape is a quite interesting issue and the PLANET-B orbit is suitable for studying the bow shock (see Fig. 3). Together with plasma measurements, we can discuss the physics operating in the Martian bow shock.

The charged-particle motion is diffusive at the Martian bow shock, and it would make an upstream region different from that at Earth. The presence of reflected ion beams characterizes the upstream region of the Earth bow shock. They become a diffuse distribution while exciting upstream waves and are eventually convected downstream. The Martian bow shock is also preceded by a foreshock, a region where the plasma and magnetic field fluctuate considerably. The spectral peak seen in the magnetic field fluctuations are very close to the proton gyro-frequency (Russell et al., 1990). This makes a clear contrast in the upstream waves in the Earth's bow shock, where the dominant fluctuations are in a frequency range below the gyro-frequency of the proton (e.g., Fairfield, 1969). These observations suggest that the presence of pick-up ions in the upstream region affects the excitation of upstream waves in Mars. The generation of reflected ion beams might be insignificant because of the small size of the bow shock. PLANET-B will cover a large area of the upstream region (see Fig. 3), and high-time resolution measurements of the magnetic field will enable us to investigate the physical processes taking place there.

\section{Instrumentation}

The subsystem for magnetic field measurements (MGF) consists of a three-axis fluxgate magnetometer sensor and electronics. A fluxgate magnetometer with a ring core geometry is used in this experiment because of its robustness and low power. The sensor, mounted at the end of a deployable mast (MST-M), is located at a distance of $6 \mathrm{~m}$ from the spacecraft spin axis. The design concept of the sensorelectronics system is basically the same as those used in the
Table 2. Data compression modes for magnetic field measurements.

\begin{tabular}{cc}
\hline Telemetry bit rate & Data resolution after compression \\
\hline $65536 \mathrm{bps}$ & $\sim 32 \mathrm{~Hz} ; 15$ bit resolution \\
$32768 \mathrm{bps}$ & $\sim 32 \mathrm{~Hz} ; 15$ bit resolution \\
$16384 \mathrm{bps}$ & $\sim 16 \mathrm{~Hz} ; 15$ bit resolution \\
$8192 \mathrm{bps}$ & $\sim 8 \mathrm{~Hz} ; 15$ bit resolution \\
$4096 \mathrm{bps}$ & $\sim 4 \mathrm{~Hz} ; 12$ bit resolution \\
$2048 \mathrm{bps}$ & $\sim 2 \mathrm{~Hz} ; 12$ bit resolution \\
$1024 \mathrm{bps}$ & $\sim 1 \mathrm{~Hz} ; 12$ bit resolution \\
$512 \mathrm{bps}$ & $\sim 1 \mathrm{~Hz} ; 10$ to 12 bit resolution \\
$256 \mathrm{bps}$ & $\sim 1 \mathrm{~Hz} ; 10$ to 12 bit resolution \\
$128 \mathrm{bps}$ & $\sim 16 \mathrm{sec} ; 15$ bit resolution \\
$64 \mathrm{bps}$ & $\sim 32 \mathrm{sec} ; 15$ bit resolution \\
\hline
\end{tabular}

successful AKEBONO and GEOTAIL missions (Fukunishi et al., 1990; Kokubun et al., 1994).

The analog part of the MGF instrument is of standard design and consists of an amplifier, a filter, a phase sensitive detector, an integrator, and a voltage-current converter. Four dynamic ranges from \pm 16 to $\pm 65536 \mathrm{nT}$ are prepared and are switched automatically by the onboard CPU. The automatic operation of range control can be interrupted at any time by a ground command. The sampling for magnetic field data is synchronized with the spacecraft spin motion. A 16-bit A/D converter (15-bit accuracy) is adopted to satisfy the data resolution from the scientific requirement. The sampling rate is 256 vectors every spin (about 32 vectors/second). Table 1 summarizes the characteristics of the fluxgate magnetometer onboard PLANET-B.

Sampled data are compiled according to the spacecraft data mode and bit rate. The onboard data compilation mode has been designed so as to expect a maximum science output. The data compilation bit rate varies to a quite large extent from $65.536 \mathrm{kbps}$ to $64 \mathrm{bps}$, while the telemetry bit rate varies from 32.768 to $2.048 \mathrm{kbps}$ depending on the Mars-Earth distance. The science data acquired will be recorded by the onboard data recorder and reproduced when the ground station links with the spacecraft. In the low altitude around the periapsis, the data compilation bit rate is planned to be the highest $(65.536 \mathrm{kbps})$ and the MGF sends the highest time resolution data of about 32 vectors per second. The compilation bit rate in other areas will depend on the telemetry bit rate. The bit rate of $256 \mathrm{bps}$ or larger is typical during observation in higher altitudes. One second time resolution, at least, is established for magnetic field data even when the bit rate is as low as $256 \mathrm{bps}$, to make it possible for us to study upstream wave phenomena. Table 2 
summarizes the data compression strategy for the magnetic field experiment.

A special effort has been made to reduce the total mass of the MGF system. This is one of the most challenging items in the design phase of each subsystem to realize the PLANET$\mathrm{B}$ mission. In the MGF instrument, HIC technology has been introduced to the analog electronics component. Gate arrays and surface-mount-type devices are adopted for digital components. The subsystem weight becomes about $1 / 3$ in comparison with a similar type of magnetometer previously manufactured. To reduce the total mass even more, a great challenge was introduced, that is, the subsystem power unit and the telemetry/command I/F control unit are shared with UVS (ultraviolet spectrometer), PET (electron temperature probe), and MST (extensible mast) instruments. The electronic boards for these subsystems are installed in one unit (MPM-E). A common CPU controls both telemetry and command interface. It also gathers raw data from MGF, PET, and UVS and edits them to send through the telemetry.

During the ground test of the spacecraft, significant efforts have been made to reduce spacecraft-generated magnetic fields through the cooperation of many people, including members of the PWA team and other scientific and engineering teams. Special care has been taken in the design of each subsystem, such as the wiring inside the spacecraft and the layout of the solar cell arrays. Care was taken during the design phase to minimize the use of sources of stray fields such as permanent magnets and electromagnets. When an unavoidable stray field was found in the ground test, a magnet was used to cancel it. Currently, we can confirm that the bias field from the spacecraft would be smaller than $1 \mathrm{nT}$ at the location of the magnetometer sensor. The final resolution will be obtained from the ground test which begin in October 1997.

\section{Summary}

Some scientific topics relating to magnetic field measurements onboard PLANET-B are briefly described and the specifications of the magnetic field instrument onboard PLANET-B is presented. PLANET-B will be inserted into a highly elliptical orbit around Mars in October 1999. A planned periapsis altitude of $150 \mathrm{~km}$ and an apoapsis of $15 \mathrm{R}_{\mathrm{M}}$ would enable us to observe magnetic environments in various regions around the planet Mars. One of the most important objectives is to resolve the controversies concerning the magnitude of the magnetic moment of Mars. The combination of measurements with other instruments onboard PLANET-B will give us important information on unsolved problems such as pressure balance in the dayside ionosphere and acceleration processes for ionospheric ions.

Acknowledgments. The authors thank the PLANET-B project teams in making special efforts to realize a magnetically clean spacecraft. They are indebted to the other co-investigators of the magnetic field team concerned with the ground test. The magnetic field instrument (MGF) is manufactured by Mesei Electric Company, and the extendible mast is manufactured by the Japan Aircraft Company.

\section{References}

Dolginov, Sh. Sh., Comments on "The magnetic field of Mars; Implications from gas dynamic modeling" by Russell et al., J. Geophys. Res., 91, 12143, 1986.

Dolginov, Sh. Sh., Ye. G. Yeroshenko, and L. N. Zhuzgov, Magnetic field in the very close neighborhood of Mars according to data from the Mars 2 and Mars 3 spacecraft, J. Geophys. Res., 78, 4779-4786, 1973.

Dolginov, Sh. Sh., Ye. G. Yeroshenko, and L. N. Zhuzgov, The magnetic field of Mars according to the data from the Mars 3 and Mars 5, J. Geophys. Res., 81, 3353-3362, 1976.

Fairfield, D. H., Bow shock associated waves observed in the far upstream interplanetary medium, J. Geophys. Res., 74, 3541, 1969.

Fukunishi, H., R. Fujii, S. Kokubun, K. Hayashi, F. Tohyama, Y. Tonegawa, S. Okano, M. Sugiura, K. Yumoto, I. Aoyama, T. Sakurai, T. Saito, T. Iijima, A. Nishida, and M. Natori, Magnetic field observations on the AKEBONO (EXOS-D) satellite, J. Geomag. Geoelectr., 42, 385-409, 1990.

Kokubun, S., T. Yamamoto, M. H. Acuna, K. Hayashi, K. Shiokawa, and H. Kawano, The GEOTAIL magnetic field experiment, J. Geomag. Geoelectr., 46, 7-21, 1994.

Luhmann, J. G. and L. H. Brace, Near-Mars space, Rev. Geophys., 22, 121140, 1991.

Russell, C. T., The magnetic file of Mars: Mars 3 evidence reexamined, Geophys. Res. Lett., 5, 81-84, 1978a.

Russell, C. T., The magnetic file of Mars: Mars 5 evidence reexamined, Geophys. Res. Lett., 5, 85-88, 1978 b.

Russell, C. T., Magnetic fields of the terrestrial planets, J. Geophys. Res., 98, 18,681-18,695, 1993.

Russell, C. T., J. G. Luhmann, J. R. Spreiter, and S. S. Stahara, The magnetic field of Mars; Implications from gas dynamic modeling, $J$. Geophys. Res., 89, 2997-3003, 1984.

Russell, C. T., J. G. Luhmann, K. Schwingenschuh, W. Riedler, and Ye. Yeroshenko, Upstream waves at Mars: PHOBOS observations, Geophys. Res. Lett., 17, 897-900, 1990.

Russell, C. T., M. Ong, J. G. Luhmann, K. Schwingenschuh, W. Riedler, and Ye. Yeroshenko, Bow shocks and magnetotails of Venus and Mars: A comparison, Adv. Space Res., 12, (9)163-(9)167, 1992.

Russell, C. T., T. Mulligan, M. Delva, T.L.Zhang, and K. Schwingenschuh, A simple test of the induced nature of the Martian tail, Planet. Space Sci., 43, 875-879, 1995.

Sauer, K., Th. Roatsch, U. Motschmann, D. Mohlmann, K. Schwingenschuh, and W. Riedler, Plasma boundaries at Mars discovered by the Phobos 2 magnetometers, Ann. Geophys., 8, 661-670, 1990.

Schwingenschuh, K., W. Riedler, H. Lichtenegger, Ye. Yeroshenko, K. Sauer, J. G. Luhmann, M. Ong, and C. T. Russell, Martian bow shock: PHOBOS observations, Geophys. Res. Lett., 17, 889-892, 1990.

Shinagawa, H. and T. E. Cravens, A One-dimensional multispecies magnetohydromagnetic model for the dayside ionosphere of Mars, $J$. Geophys. Res., 94, 6506-6516, 1989.

Showstack, R., Mars has crustal, complex magnetic field, EOS, 78, 429, 1997.

Slavin, J. A., K. Schwingenschuh, W. Riedler, and Ye. Yeroshenko, The solar wind interaction with Mars: Mariner 4, Mars 2, Mars 3, Mars 5, and Phobos 2 observations of bow shock position and shape, J. Geophys. Res., 96, 11235-11241, 1991.

Yeroshenko, Ye., W. Riedler, K. Schwingenschuh, J. G. Luhmann, M. Ong, and C. T. Russell, The magnetotail of Mars: PHOBOS observations, Geophys. Res. Lett., 17, 885-888, 1990.

Zhang, M. H. G. and J. G. Luhmann, Comparisons of peak ionosphere pressures at Mars and Venus with incident solar wind dynamic pressure, J. Geophys. Res., 97, 1017-1025, 1992.

Zhang, M. H. G., J. G. Luhmann, A. J. Kliore, and J. Kim, A post-pioneer Venus reassesment of the Martian dayside ionosphere as observed by radio occultation methods, J. Geophys. Res., 95, 14829, 1990.

Zhang, T.-L., J. G. Luhmann, and C. T. Russell, The solar cycle dependence of the location and shape of the Venus bow shock, J. Geophys. Res., 95, 14961-14967, 1990.

T. Yamamoto and A. Matsuoka (e-mail: matsuoka@stp.isas.ac.jp) 